\title{
CONHECIMENTO DA RADIOPROTEÇÃO EM UNIDADE ORTOPÉDICA DA CIDADE DE CERES-GO
}

\author{
KNOWLEDGE OF RADIOPROTECTION IN AN ORTHOPEDIC UNIT OF THE CITY OF \\ CERES-GO
}

\author{
Janayna Vaz da Silva Godoy \\ Discente do Curso de Radiologia da Faculdade Evangélica de Ceres, Goiás. \\ E-mail: janaynaavaz@gmail.com \\ Jander de Souza Godoy \\ Discente do Curso de Radiologia da Faculdade Evangélica de Ceres, Goiás. \\ E-mail: jandergodoy@msn.com
}

Carla Danielle Dias Costa

Docente da Faculdade Evangélica de Ceres.

Endereço para correspondência. Av. Brasil, s/n, Qd 13, Morada Verde, Ceres-Goiás, Brasil. CEP: 76300-000.

Fone: (62) 3323-1040 E-mail: carladaniellebm@ gmail.com

\section{RESUMO}

INTRODUÇÃO: A Radioproteção é uma medida eficaz na proteção dos profissionais de radiologia no exercício de suas funções, ela é uma prática de extrema importância, porém muitos profissionais e unidades radiológicas não seguem adequadamente todos os requisitos estabelecidos pela Portaria $n^{\circ}$ 453/1998. OBJETIVO: O objetivo deste estudo foi identificar a adequação de um hospital ortopédico às diretrizes da radioproteção, assim como conhecer a prática da radioproteção entre os profissionais da área de radiologia e registrar os Equipamentos de Proteção Individual e Equipamentos de Proteção Coletiva disponíveis no local. METODOLOGIA: Esta é uma pesquisa observacional, transversal de caráter descritivo, realizada em uma Unidade Hospitalar Ortopédica, localizada na cidade de CeresGO. Foi preenchido um checklist contendo 4 campos de avaliação das seguintes categorias: equipamentos de proteção individual; equipamentos de proteção individual (EPI) de uso do profissional, disponível na sala de Raios X; equipamentos de proteção coletiva (EPC), requisitos operacionais e legais, a fim de verificar a adequação à legislação. RESULTADOS E DISCUSSÃO: Por meio da observação foi possível identificar que a unidade ortopédica vistoriada continha apenas uma sala de Raios - X, onde eram realizados os procedimentos radiológicos. Durante a visita foi observado à disponibilização de EPIs, como aventais, luvas de proteção, óculos plumbíferos e EPCs sendo eles vidro plumbífero, parede de chumbo, porta de aço. Constatou - se que o hospital oferece todos os equipamentos necessários de proteção (EPIs e EPCs) tanto aos profissionais de radiologia e pacientes primando pela segurança e proteção de todos os envolvidos, no entanto os mesmos não são utilizados adequadamente em determinados procedimentos. CONCLUSÃO: Através do estudo realizado na Unidade Hospitalar Ortopédica foi possível observar que a unidade disponibiliza os EPIs e EPCs aos profissionais e pacientes, fornece capacitações constantes aos seus profissionais, com o objetivo de mantê-los sempre preparados para o exercício das atividades. No entanto a mesma atende parcialmente aos requisitos da Portaria $n^{\circ}$ 453/1998 que regulamenta a atividade de radioproteção. 
Palavras-Chave: Radiologia. Radiação. Radioproteção.

ABSTRACT

INTRODUCTION: Radioprotection is one of the effective functions in radioprotection of radiology is not an exercise of its functions, it is a practice of extreme gum, many years and its limits of support are not defined by Ordinance $n^{\circ}$ 453/1998. OBJECTIVE: The objective of this study was to identify the adequacy of an orthopedic hospital to the guidelines of radioprotection, as well as to know the practice of radioprotection among professionals in the radiology area and record of Individual Protection Equipment and Collective Protection Equipment available on the spot. METHODOLOGY: This is a cross-sectional, observational, descriptive study performed at an Orthopedic Hospital Unit located in the city of Ceres-GO. A checklist containing 4 assessment fields of the following categories was completed: individual protection equipment; personal protective equipment (PPE) for professional use, available in the X-ray room; collective protection equipment (EPC), operational and legal requirements, in order to verify compliance with legislation. RESULTS AND DISCUSSION: Through observation it was possible to identify that the orthopedic unit surveyed contained only one $\mathrm{X}$ - ray room, where the radiological procedures were performed. During the visit it was observed the availability of PPE, such as aprons, protective gloves, goggles and EPCs, such as lead glass, lead wall, steel door. It was verified that the hospital offers all the necessary equipment of protection (PPEs and EPCs) to radiology professionals and patients for the safety and protection of all involved, however they are not used properly in certain procedures. CONCLUSION: Through the study performed at the Orthopedic Hospital Unit, it was possible to observe that the unit provides the EPIs and EPCs to professionals and patients, it provides constant training to its professionals, with the objective of keeping them always prepared for the exercise of activities. However, it partially meets the requirements of Administrative Rule 453/1998, which regulates the radioprotection activity.

Key-words: Radiology. Radiation. Radioprotection. 


\section{INTRODUÇÃO}

As radiações se distinguem quanto aos seus efeitos ao interagirem com a matéria, classificando-se em ionizantes ou não ionizantes. Segundo Bushong (2010), compreende-se por radiação ionizante a radiação capaz de remover um elétron do orbital do átomo com o qual interage. Este tipo de interação entre a radiação e a matéria é denominado de ionização.

A radiação ultravioleta, assim como a luz visível, infravermelho, radiofrequência (RF), frequências extremamente baixas são consideradas radiações não ionizantes, por não terem energia suficiente para arrancar um elétron dos principais átomos que constituem o corpo humano, como o hidrogênio, oxigênio, carbono e nitrogênio, além de ter pouca absorção no corpo humano (OKUNO, 2013).

A radiação ao entrar em contato com o organismo desencadeia reações nos indivíduos, que são caracterizados como efeitos biológicos da radiação e podem ser divididos em dois grupos: efeitos determinísticos e efeitos estocásticos (FREITAS; ROSA; SOUZA, 2004). Efeitos determinísticos são aqueles que se manifestam nos organismos atingidos após a exposição do mesmo a uma determinada dose, a qual supera o limiar de regeneração celular. São os efeitos agudos da radiação, tais como: eritema cutâneo, desenvolvimento de processos fibróticos. Tais efeitos possuem um tempo de latência que varia de horas até dias (PASLER; VISSER, 2005).

Os efeitos estocásticos ocorrem quando a probabilidade de surgimento de efeitos biológicos - não o grau de severidade - está em função da dose administrada. Como exemplo pode-se citar os tumores malignos induzidos por radiação e as alterações genéticas. Os efeitos estocásticos podem apresentar um tempo de latência de vários anos, são graves e para os mesmos não existe uma dose limiar de desencadeamento, a probabilidade de desenvolvimento desses efeitos aumenta com o aumento da dose (PASLER; VISSER, 2005).

A partir do descobrimento dos raios $\mathrm{X}$ e da radioatividade, iniciou-se o uso desregrado da radiação por profissionais de saúde, que começaram a compreender o seu potencial em remover pintas, matar células. No entanto, somente 30 anos após o descobrimento dos raios X é que foi criada a Comissão Internacional de Unidades e Medições de Radiação, com o intuito de constituir grandezas e unidades de física das radiações, critérios de medidas, métodos de comparação.

Em seguida foi estabelecida a Comissão Internacional de Proteção Radiológica, com a finalidade de elaborar normas de proteção radiológica para criar limites de exposição à 
radiação ionizante para os profissionais de radiologia e para público em geral. No âmbito nacional, o órgão que regulamenta o uso de radiações é a Comissão Nacional de Energia Nuclear (CNEN) (OKUNO, 2013).

A radioproteção trata-se de uma prática de proteção aos profissionais que atuam na área de radiologia e que estão diariamente expostas as radiações ionizantes. Tendo em vista todos os riscos característicos desta atividade, no intuito de conscientizar os profissionais da área da importância de exercer sua atividade de forma consciente e responsável (BRAND; FONTANA; SANTOS, 2011).

O emprego correto da radioproteção é a maneira mais segura de desfrutar dos benefícios gerados pela radiação ionizante com a atenuação e em alguns casos com a isenção dos males causados por esse fenômeno. Para isso, é necessário seguir todas as normas em vigor, estabelecidas e fiscalizadas pelos órgãos competentes - CNEN e a Agência Nacional de Vigilância Sanitária (ANVISA) - e utilizar com rigor todos os equipamentos de proteção individual e coletiva (SAVAREGO, DAMAS, 2007).

A ANVISA destaca que os organismos nacionais e internacionais que regulam as normas de proteção radiológica estabeleceram princípios para que todos os profissionais que lidam com radiações ionizantes, paciente e público em geral, possam conviver de uma forma segura e harmoniosa com essa forma de energia. Os princípios são: justificativa, otimização e limitação de dose e estão definidos na Portaria Federal n 453, de 2 de junho de 1998, da ANVISA (BRASIL, 1998).

A Norma Regulamentadora (NR6), prevê o uso necessário e obrigatório do uso de Equipamentos de Proteção Individual (EPI) nas atividades laborais. São considerados EPIs todos os dispositivos ou produtos de uso individual utilizado pelo trabalhador, destinado à proteção de riscos suscetíveis de ameaçar a segurança e a saúde no trabalho (BRASIL, 1978).

$\mathrm{Na}$ utilização dos raios $\mathrm{X}$ nos procedimentos em radiodiagnóstico para atingir o objetivo radiológico, deve-se ter em mente que todo meio de proteção radiológica deve ser utilizado para que as doses, principalmente nos profissionais de radiologia, sejam tão baixas quanto possíveis. Deve-se implementar ações e práticas necessárias para minimizar a contribuição de erros humanos que levem à ocorrência de exposições acidentais. Mantendo as instalações e os equipamentos de raios-X nas condições exigidas pela Portaria 453, devendo prover serviço adequado de manutenção periódica. É preciso evitar a realização de exposições desnecessárias e em hipótese alguma, substituir a observação às práticas de proteção e segurança da radioproteção (BRASIL, 1998). 
O princípio de ALARA - "As Low As Reasonably Achievable”, estabelece em sua filosofia básica a redução da exposição do indivíduo ocupacionalmente exposto aos menores índices possíveis de dose, as relações de dose e sua propagação em ambientes. Este princípio baseia-se na identificação, avaliação e análise para implementação de medidas de controle da radiação no intuito de manter os trabalhadores dentro de parâmetros aceitáveis tão baixos quanto possível, ou seja, mesmo dentro de limites de exposição convencionados. A filosofia de ALARA busca a implementação de ações para a redução das doses de exposição as quais os indivíduos expostos se submetem (DIAS, 2015).

Os três grandes fatores para a redução das exposições as radiações externas e manutenção de doses na filosofia de ALARA são: Tempo, blindagem e distância. Estes se referem à redução do tempo de exposição ao mínimo necessário, para uma determinada técnica de exames. Esta é a maneira mais prática para se reduzir a exposição à radiação ionizante e quanto mais distante da fonte de radiação, menor a intensidade do feixe. Realizar o exame no menor tempo possível sem perder o objetivo clínico do tratamento. Quanto maior a distância menor a exposição à radiação no paciente, e com uso de barreiras artificiais entre a fonte e o indivíduo (SILVA, 2013).

Embora a radioproteção seja uma prática de extrema importância, muitos profissionais e unidades radiológicas não seguem adequadamente todos os requisitos estabelecidos por lei. Tal descumprimento faz com que surjam situações preocupantes como o risco de contaminação pelos efeitos radioativos na exposição de profissionais, pacientes e do meio ambiente (BRAND; FONTANA; SANTOS, 2011).

O objetivo deste estudo foi identificar a adequação das diretrizes da radioproteção em uma unidade hospitalar ortopédica, assim como conhecer a prática da radioproteção entre os profissionais da área de radiologia e registrar os equipamentos de proteção individual (EPI) e os equipamentos de proteção coletiva (EPC) disponíveis nesta unidade.

\section{METODOLOGIA}

O presente artigo trata-se de uma pesquisa transversal, observacional e descritiva, analisando os itens de Radioproteção da área onde são realizados os procedimentos referentes aos exames de Raios- X, em um Hospital Ortopédico do município de Ceres- Goiás. A pesquisa foi realizada no mês de setembro de 2017, por meio de um instrumento de pesquisa, checklist. Para a realização do trabalho, obteve-se a autorização da chefia da unidade, como se observa no Apêndice 1.

REFACER v 7. , n. 1, 2018. ISSN - 2317-1367 


\section{Coleta de dados}

O instrumento de pesquisa utilizado foi um checklist, contendo 4 campos de avaliação das seguintes categorias: Equipamentos de proteção individual (EPI) (disponíveis na sala de raio x); Equipamentos de proteção individual (EPI) (uso do profissional); equipamentos de proteção coletiva (EPC), requisitos operacionais e legais. Para se preencher as categorias analisadas, a medida que se faziam as avalições, os campos "sim", "não" e "não informado", eram marcados. (Apêndice 2). O instrumento de pesquisa foi construído com base no estudo realizado por Burigo (2007).

No dia 23 de setembro de 2017, foi realizada uma visita a Unidade Hospitalar Ortopédica da cidade de Ceres-GO, a fim de observar a adequação da sala de procedimentos radiológicos aos itens exigidos pela legislação. O checklist foi preenchido pelos pesquisadores. Á medida que os mesmos faziam as observações dos EPIs, EPCs, documentações e certificações que estavam afixados nas paredes da unidade, os pesquisadores assinalavam os campos disponíveis. Caso algum dos itens referentes aos requisitos operacionais e legais não estivessem de maneira visível, os mesmos foram solicitados à secretaria da unidade.

A análise dos dados da entrevista foi realizada por meio de estatística descritiva simples.

\section{RESULTADOS E DISCUSSÃO}

Através da pesquisa realizada na unidade ortopédica da cidade de Ceres - GO constatou-se que a mesma possui apenas uma sala de Raios $X$, onde são realizados os procedimentos radiológicos. A mesma possui 3 profissionais técnicos de Radiologia e 2 estagiários atuando em regime de plantão. O artigo 14 da Lei 7.394/85 regulamenta a profissão de Técnico em Radiologia e prevê que a jornada de trabalho dos profissionais de radiologia deve ser de 24 (vinte e quatro) horas semanais. Sendo assim, observou-se que a quantidade de profissionais disponíveis na unidade, está adequada ao proposto pela legislação (BRASIL, 1985).

Durante a visita observou-se os EPIs radiológicos disponíveis na sala de Raios X, para serem utilizados pelos profissionais e pacientes. No ambiente foram encontrados instrumentos como, avental de chumbo; luva plumbífera; óculos plumbífero; protetor de gônadas; protetor de tireóide e dosímetro. O uso destes equipamentos de proteção por profissionais e pacientes é de extrema importância, visto que, a prevenção em qualquer 
atividade sujeita à radiação ionizante é fundamental. Uma vez que, as radiações não são percebidas pelos sentidos humanos, independentemente do nível de radiação emitida durante as atividades desempenhadas na radiologia diagnóstica, visto que os danos biológicos existem (MASTROENI, 2004).

Em relação aos EPIs radiológicos, como aventais, saias de proteção, protetores abdominais, luvas de proteção, mangas, proteção para membros inferiores, protetor de gônadas para pacientes masculinos, óculos plumbíferos e protetores de tireóide, observou-se que o uso destes equipamentos é constante em todos os procedimentos radiológicos. Estes equipamentos de proteção devem ser usados no trabalho direto à fonte de radiação, pois a radiação ionizante pode atuar de forma cumulativa, causando danos à saúde. Dessa forma o uso dos EPIs radiológicos é imprescindível na promoção da saúde dos trabalhadores que exercem as atividades em radiologia (FERNANDES, 2005; BRASIL, 1978).

Quanto aos EPIs tradicionais (luvas, máscaras, jalecos, toucas), os mesmos estavam presentes na sala de raios-X, no entanto os profissionais não fizeram o uso destes materiais em todos os momentos durante a observação. Foi identificado que em atendimentos aos pacientes que não apresentavam sangramentos visíveis ou fratura exposta, os profissionais não fizeram uso de luvas e máscaras durante o procedimento, talvez pelo fato de acreditarem que nesses casos não estivessem suscetíveis a nenhum risco.

Os equipamentos de proteção individual são utilizados na prevenção do profissional aos riscos que atingem a saúde do mesmo durante a execução da atividade laboral. O uso correto dos EPIs traz numerosos benefícios aos trabalhadores. As luvas de procedimentos são indicadas independentemente da possibilidade de contato com sangue e secreções, com mucosa ou pele não íntegra do paciente; já as máscaras, devem ser empregadas na realização de procedimentos em que haja chance de contato com sangue ou outras secreções (GONÇALVES; GONÇALVES; GONÇALVES, 2015). Apesar dos profissionais de radiologia da Unidade Hospitalar pesquisada, não estarem a todo o momento em contato com sangue, existem a chance de contato com gotículas de espirros, saliva dos pacientes e acompanhantes. A falta do EPI correto pode estar colocando em risco a saúde desses profissionais.

Os Equipamentos de Proteção Coletiva (EPC) abrangem toda estrutura fixa ou móvel de abrangência coletiva, destinando-se a preservar a integridade física e a saúde dos trabalhadores, usuários e terceiros que estão em contato ou próximos à área radiológica. (GONÇALVES; GONÇALVES; GONÇALVES, 2015). 
Durante a vistoria dos EPCs instalados na sala de raios $\mathrm{X}$ e dependências, verificou-se que esses equipamentos são usados de acordo com a Portaria $n^{\circ}$ 453/1998, como é o caso do vidro plumbífero; visor plumbífero; parede de chumbo; porta de aço; biombo; placas de advertência; sinalização luminosa; dosimetria, tudo para garantir a segurança dos profissionais e pacientes expostos à radiação. É fundamental que os profissionais estejam atentos ao que a regulamentação prevê, uma vez que, toda a segurança e proteção da unidade dependem do trabalho responsável e consciente dos profissionais de radiologia.

Os EPCs são de grande importância para a unidade, visto que quando bem aplicados, barram a propagação da radiação para outras áreas, impossibilitando que outros profissionais sejam atingidos. Tendo em vista que os EPCs são utilizados com o intuito de diminuir a exposição de trabalhadores e pacientes aos riscos e em casos de acidentes diminuírem as consequências danosas (MÔNICO et al., 2014).

As placas luminosas de sinalização tratam se de um instrumento que serve de alerta, dando informações aos pacientes, acompanhantes e demais profissionais, quanto aos perigos existentes no local. O dosímetro é um instrumento essencial no exercício da atividade de radiologia, visto que o mesmo avalia a quantidade de radiação no presente local, de modo que caso o mesmo acuse uma quantidade alta de radiação é preciso que medidas sejam tomadas para que esses níveis sejam controlados, para evitar que profissionais e pacientes corram riscos (MELO et al., 2015).

Também foram observados itens como a existência de responsável técnico, educação continuada, realização de exames periódicos entre os profissionais, licenciamento ambiental e vistoria (físico) e verificou-se que esses requisitos estão de acordo com a regulamentação de que trata a Portaria 453, de 1998, ficando evidente que a Unidade Ortopédica corrobora com o que está estabelecido na lei. Já quanto aos requisitos operacionais e legais no que se refere à manutenção dos equipamentos, quando verificadas tais informações não foi possível avaliar o presente item, pois a documentação não estava disponível.

A função do responsável técnico é exercer a responsabilidade técnica e organizacional da unidade, desempenhando atividades como observar com rigor as normas legais de proteção contra as irradiações ionizantes no desempenho de suas atividades profissionais, com o objetivo de resguardar sua saúde e dos clientes. Sendo de sua responsabilidade a proteção das pessoas nas áreas irradiadas, bem como a utilização correta dos equipamentos de segurança (CONTER, 2017). 
A educação continuada na unidade pesquisada era realizada frequentemente, de modo que cursos de capacitação eram oferecidos pela unidade, aplicados por profissionais da saúde, como médicos, enfermeiros, radiologistas, dependendo do tema a ser abordado, a todos os profissionais da região de Ceres-GO. A Educação Continuada possibilita o aprendizado e a capacitação do profissional, de modo que os conteúdos e objetivos atendam a realidade, o diaa-dia do trabalho e as necessidades dos profissionais no ambiente laboral (SILVA; SEIFFERT, 2009).

Os exames médicos periódicos são fundamentais para avaliação do estado de saúde dos trabalhadores, e tem como um dos objetivos orientá-los quanto aos fatores de risco, no caso de profissionais em radiologia essa atenção é ainda mais imprescindível, pois se tratam de profissionais que lidam diariamente com a radiação. O Programa de Controle Médico de Saúde Ocupacional (PCMSO) prevê a obrigatoriedade de se realizar exame médico admissional e periódico para os profissionais expostos à radiação, assim como o exame de laboratório mínimo, como o hemograma completo e a contagem de plaquetas semestralmente, visando resguardar esses profissionais de problemas de saúde (SOUZA; SOARES, 2008).

A execução deste estudo se mostrou significativa, embora a metodologia utilizada foi de caráter observatório. Posteriormente novos estudos empregando a metodologia baseada em uma entrevista com os profissionais da unidade poderiam ser realizados com os profissionais da unidade (técnicos, tecnólogos, enfermeiros, secretárias) no intuito de conhecer a percepção dos mesmos, sobre a radioproteção e assim substanciar os resultados encontrados no presente trabalho.

\section{CONCLUSÃO}

Os equipamentos de proteção individuais ou coletivos devem fazer parte contínua no exercício da profissão do radiologista, pois eles são essenciais para a segurança e proteção dos profissionais e pacientes em contato com a radiação. Foi observado que o hospital oferece todos os equipamentos necessários de proteção (EPIs e EPCs) tanto aos profissionais de radiologia e pacientes primando pela segurança e proteção de todos os envolvidos.

Identificou-se que todos os equipamentos de proteção individual e coletiva estão disponíveis na unidade pesquisada, sendo utilizados pelos profissionais, embora não sejam utilizados por todos os profissionais em alguns tipos de procedimentos.

A unidade Ortopédica demonstrou fornecer capacitações constantes aos seus profissionais, no intuito de mantê-los sempre preparados para o exercício dos procedimentos 
radiológicos. No entanto atende parcialmente as exigências da portaria 453 que regulamenta a radioproteção, de modo que os documentos referentes aos requisitos operacionais e legais no que diz respeito à manutenção dos equipamentos, não estavam públicos para a população em geral.

\section{REFERÊNCIAS}

BRAND, C. I; FONTANA, R.T; SANTOS, A.V.S. A saúde do trabalhador em radiologia: algumas considerações. Texto contexto - enfermagem. vol.20 no.1 Florianópolis Jan./Mar. 2011. Disponível em<http://www.scielo.br/scielo.php?script=sci_arttext\&pid=S0104$07072011000100008>$ Acesso em: 25 nov. 2017.

BRASIL. Diretrizes de proteção radiológica em radiodiagnóstico médico e odontológico. Portaria n. 453 (1998). Brasília, DF: ANVISA, 1998.

BRASIL. Aprova as Normas Regulamentadoras - NR - do Capítulo V, Título II, da Consolidação das Leis do Trabalho, relativas a Segurança e Medicina do Trabalho. Portaria n. ${ }^{\circ} 3.214$ de 08 de junho de 1978. Disponível em<http://www.camara.gov.br/sileg/integras/839945.pdf> Acesso em: 26 nov. 2017.

BRASIL. Regula o Exercício da Profissão de Técnico em Radiologia, e dá outras providências. Lei $\mathrm{n}^{\mathrm{o}} 7.394$, de 29 de outubro de 1985. Disponível em<http://www.planalto.gov.br/ccivil_03/leis/L7394.htm>Acesso em: 26 nov. 2017.

BURIGO, R.L; BURIGO, V.V. Avaliação dos requisitos de proteção radiológica em Clínica de radiodiagnóstico. 2007.2 Disponível em<http://www.bib.unesc.net/biblioteca/sumario/000030/000030ED.pdf> Acesso em: 09 out. 2017.

BUSHONG, S.C.Ciência radiológica para tecnólogos: física, biologia e proteção. Rio de Janeiro: Elsevier, 2010.

BUSTAMANTE, M.C. A descoberta dos raios cósmicos ou o problema da ionização do ar atmosférico. Revista Brasileira Ensino Físico. vol.35 no.2 São Paulo Apr./June 2013. Disponível em< http://www.scielo.br/scielo.php?script=sci_arttext\&pid=S1806$11172013000200030>$ Acesso em: 24 nov. 2017.

CONTER- Conselho Nacional de Técnico em Radiologia. 2017. Disponível em<http://www.crtr17.gov.br/arquivos/codigo_etico.pdf> Acesso em: 24 nov.2017.

DIAS, F.A. Princípio ALARA e a Proteção Radiológica. 2015. Disponível em<http://institutodiasfilho.blogspot.com.br/2015/07/filosofia-alara-e-protecaoradiologica.html> Acesso em: 14 nov. 2017.

FERNANDES, G.S; CARVALHO, A.C.P, AZEVEDO, A.C.P. Avaliação dos riscos ocupacionais de trabalhadores de serviços de radiologia. 2005. Radiologia Brasileira [online]. 2005;38(4):279-281. Disponível 
em:http://www.scielo.br/scielo.php?script=sci_arttext\&pid=S0100-39842005000400009 > Acesso em: 26 nov. 2017.

FREITAS, A; ROSA, J.E; SOUZA, I.F.Radiologia Odontológica. 6. ed. São Paulo: Artes Médicas Ltda, 2004.

GONÇALVES, D.C; GONÇALVES, I.C; GONÇALVES, E.A. Manual de Segurança e Saúde no Trabalho. 6.ed. São Paulo: LTr, 2015.

MASTROENI, M.F. Biossegurança aplicada a laboratórios e serviços de saúde. São Paulo: Atheneu, 2004.

MELO, J.A.C; GELBCKE, F.L; HUHN, A; VARGAS, M.A.O. Processo de trabalho na enfermagem radiológica:a invisibilidade da radiação ionizante. Texto Contexto Enfermagem. Florianópolis, 2015 Jul-Set; 24(3): 801-8. Disponível em<http://www.scielo.br/pdf/tce/v24n3/pt_0104-0707-tce-24-03-00801.pdf> Acesso em: 24 nov. 2017.

MÔNICO, A; FRANÇA, K; OLIVEIRA, N; SAMPAIO, M.C. A Importância de Equipamentos de Proteção Individual. 2014. Disponível em<file:///D:/DOCUMENTOS/Downloads/587-2145-1-SM.pdf> Acesso em: 25 nov. 2017.

OKUNO, E. Efeitos biológicos das radiações ionizantes. Acidente radiológico de Goiânia. 2013. Disponível em $<$ http://www.scielo.br/scielo.php?script=sci_arttext\&pid=S010340142013000100014> Acesso em: 05 mai. 2017.

PASLER, F. A.; VISSER, H. Radiologia Odontológica: procedimentos ilustrados. Porto Alegre: Artmed, 2005.

SAVAREGO, S; DAMAS, K.F. Bases da radiologia convencional. 2 ed. - São Caetano do Sul, SP: Yendis Editora, 2007.

SILVA, Andréia Gomes da. Orientação as gestantes quanto o uso da radiação ionização nos exames de raios-x. 2013. Disponível em< http://aems.edu.br/iniciacaocientifica/download/3b89d4e4d2.pdf> Acesso em: 05 nov. 2017.

SILVA, G.M; SEIFFERT, O.M.L.B. Educação continuada em enfermagem: uma Educação continuada em enfermagem: uma proposta metodológica oposta metodológica.Revista Brasileira de Enfermagem REBEn Brasília 2009 maio-jun; 62(3): 362-6. Disponível em<http://www.scielo.br/pdf/reben/v62n3/05.pdf>Acesso em: 25 nov. 2017.

SOUZA, E; SOARES, JPM. Correlações técnicas e ocupacionais da radiologia intervencionista. Sociedade Brasileira de Angiologia e de Cirurgia Vascular. J Vasc Bras. 2008;7(4):341-350. Disponível em<http://www.scielo.br/pdf/jvb/v7n4/v7n4a09.pdf> Acesso em: 25 nov. 2017. 


\section{APÊNDICE 1}


EVANGÉLICA

DE CERES

\section{CURSO DE RADIOLOGIA}

TERMO DE AUTORIZAÇÃO DE COLETA DE DADOS

Prezado (a) Sr. (a)

Aline Rabelo de Meneses

Diretora Geral do Hospital Ortopédico de Ceres

Eu, Janayna Vaz da Silva Godoy, matriculado (a) 39611501 no curso Superior de Tecnólogos em Radiologia da Faculdade Evangélica de Ceres, sob a orientação da professora Me. Carla Danielle Dias Costa vem solicitar a V. Sa. a autorização para coleta de dados nessa instituição, com a finalidade de realizar a pesquisa de iniciação científica intitulada: Conhecimento da Radioproteção em Unidade Ortopédica da Cidade de Ceres-GO, cujo objetivo: Identificar a adequação das diretrizes da radioproteção em uma unidade hospitalar ortopédica. A coleta de dados ocorrerá mediante a utilização dos EPI's e EPC's. Igualmente, assumo o compromisso de utilizar os dados obtidos somente para fins científicos, bem como de disponibilizar os resultados obtidos para esta instituição. Agradecemos antecipadamente e esperamos contar com a sua colaboração.

Atenciosamente,

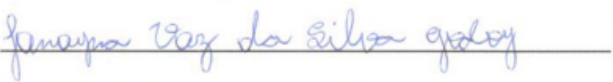

Janayna Vaz da Silva Godoy

Eu, Carla Danielle Dias Costa, responsabilizo-me pelo trabalho científico de Janayna Vaz da Silva Godoy

larla Jamielle vias losta

Carla Danielle Dias Costa

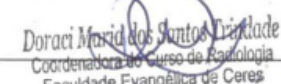

Faculdade Evangelica do Ceres

Doraci Santos Trindade

Coordenadora do Curso Superior de Tecnólogos em Radiologia

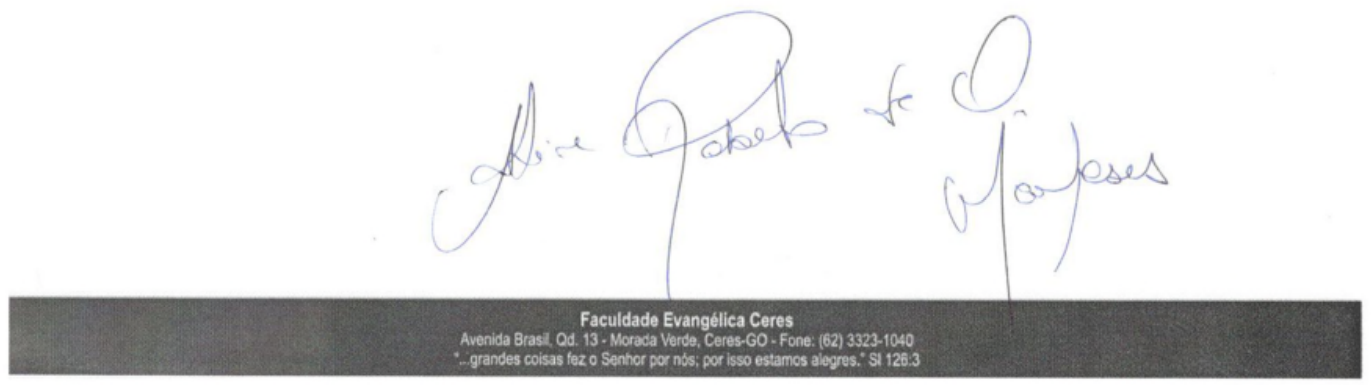

REFACER v 7. , n. 1, 2018. ISSN - 2317-1367 
CURSO DE RADIOLOGIA

\section{TERMO DE AUTORIZAÇÃO DE COLETA DE DADOS}

Prezado (a) Sr. (a)

Aline Rabelo de Meneses

Diretora Geral do Hospital Ortopédico de Ceres

Eu, Jander de Souza Godoy, matriculado (a) 811501 no curso Superior de Tecnólogos em Radiologia da Faculdade Evangélica de Ceres, sob a orientação da professora Me. Carla Danielle Dias Costa vem solicitar a V. Sa. a autorização para coleta de dados nessa instituição, com a finalidade de realizar a pesquisa de iniciação científica intitulada: Conhecimento da Radioproteção em Unidade Ortopédica da Cidade de Ceres-GO, cujo objetivo: Identificar a adequação das diretrizes da radioproteção em uma unidade hospitalar ortopédica. A coleta de dados ocorrerá mediante a utilização dos EPI's e EPC's. Igualmente, assumo o compromisso de utilizar os dados obtidos somente para fins científicos, bem como de disponibilizar os resultados obtidos para esta instituição. Agradecemos antecipadamente e esperamos contar com a sua colaboração.

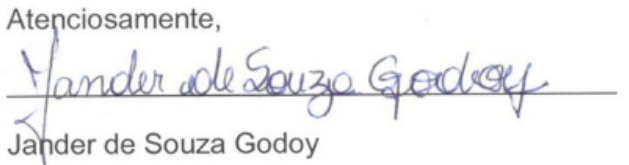

Eu, Carla Danielle Dias Costa, responsabilizo-me pelo trabalho científico de Jander de Souza Godoy

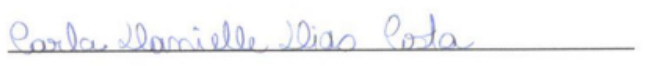

Carla Danielle Dias Costa

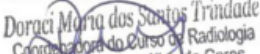

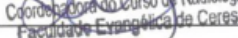

Doraci Santos Trindade

Coordenadora do Curso Superior de Tecnólogos em Radiologia

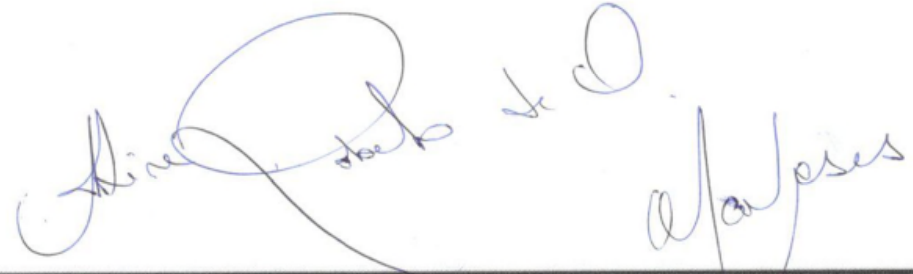




\section{APÊNDICE 2}

\section{APÊNDICE 2: CHECK LIST CONHECIMENTO DA RADIOPROTEÇÃO EM UNIDADE ORTOPÉDICA DA CIDADE DE CERES - GO.}

\begin{tabular}{|c|c|c|c|c|}
\hline \multirow[t]{2}{*}{ EQUIPE PROFISSIONAL } & Profissionais de Radiologia & \multicolumn{3}{|l|}{3 técnicos } \\
\hline & Estagiários & 2 & & \\
\hline \multirow{6}{*}{$\begin{array}{c}\text { EQUIPAMENTOS DE } \\
\text { PROTEÇÃO INDIVIDUAL } \\
\text { (EPI) } \\
\text { (Disponível na sala de Raio X) }\end{array}$} & Avental de chumbo & ( ) SIM & ( ) NÃO & ( ) NÃO INFORMADO \\
\hline & Luva Plumbífera & ( ) SIM & ( ) NÃO & ( ) NÃO INFORMADO \\
\hline & Óculos Plumbífero & ( ) SIM & ( ) NÃO & ( ) NÃO INFORMADO \\
\hline & Protetor de Gônadas & ( ) SIM & ( ) NÃO & ( ) NÃO INFORMADO \\
\hline & Protetor de Tireóide & ( ) SIM & ( ) NÃO & ( ) NÃO INFORMADO \\
\hline & Dosímetro & ( ) SIM & ( ) NÃO & ( ) NÃO INFORMADO \\
\hline \multirow{2}{*}{$\begin{array}{c}\text { EQUIPAMENTOS DE } \\
\text { PROTEÇÃO INDIVIDUAL } \\
\text { (EPI) } \\
\text { (Uso do Profissional) }\end{array}$} & Faz uso dos EPIs (Tradicionais) & ( ) SIM & ( ) NÃO & ( ) AS VEZES \\
\hline & Faz uso dos EPIs (Radiológicos) & ( ) SIM & ( ) NÃO & ( ) AS VEZES \\
\hline \multirow{8}{*}{$\begin{array}{c}\text { EQUIPAMENTOS DE } \\
\text { PROTEÇÃO COLETIVA (EPC) }\end{array}$} & Vidro Plumbífero & ( ) SIM & ( ) NÃO & ( ) NÃO INFORMADO \\
\hline & Visor Plumbífero & ( ) SIM & ( ) NÃO & ( ) NÃO INFORMADO \\
\hline & Parede de Chumbo & ( ) SIM & ( ) NÃO & ( ) NÃO INFORMADO \\
\hline & Porta de Aço & ( ) SIM & ( ) NÃO & ( ) NÃO INFORMADO \\
\hline & Biombo & ( ) SIM & ( ) NÃO & ( ) NÃO INFORMADO \\
\hline & Placas de Advertência & ( ) SIM & ( ) NÃO & ( ) NÃO INFORMADO \\
\hline & Sinalização Luminonosa & ( ) SIM & ( ) NÃO & ( ) NÃO INFORMADO \\
\hline & Dosimetria & ( ) SIM & ( ) NÃO & ( ) NÃO INFORMADO \\
\hline \multirow{7}{*}{$\begin{array}{l}\text { REQUISITOS OPERACIONAIS } \\
\text { E LEGAIS }\end{array}$} & Manutenção dos equipamentos & ( ) SIM & ( ) NÃO & ( ) NÃO INFORMADO \\
\hline & Responsável Técnico & ( ) SIM & ( ) NÃO & ( ) NÃO INFORMADO \\
\hline & Educação Continuada & ( ) SIM & ( ) NÃO & ( ) NÃO INFORMADO \\
\hline & Exames periódicos (Profissionais) & ( ) SIM & ( ) NÃO & ( ) NÃO INFORMADO \\
\hline & Licenciamento Ambiental & ( ) SIM & ( ) NÃ̃ & ( ) NÃO INFORMADO \\
\hline & Vistoria (FÍSICO) & ( ) SIM & ( ) NÃO & ( ) NÃO INFORMADO \\
\hline & $\begin{array}{l}\text { Armazenamento de filmes } \\
\text { radiográficos }\end{array}$ & ( ) SIM & ( ) NÃO & ( ) NÃO INFORMADO \\
\hline
\end{tabular}

